\title{
THE RELAPSING COURSE OF CERTAIN MENINGIOMAS IN RELATION TO PREGNANCY AND MENSTRUATION*
}

\author{
BY \\ EDWIN R. BICKERSTAFF, J. M. SMALL, and I. A. GUEST \\ From the Midland Centre for Neurosurgery, Smethwick, Birmingham
}

Intracranial tumours not uncommonly show their first symptoms, or a rapid increase in their symptoms, during a pregnancy. This occurs more frequently than can be regarded as purely coincidental, and applies to all types of tumour-extrinsic and intrinsic. On questioning a number of medical men it was evident that a popular belief is held that tumours of the pituitary are particularly likely to behave in this manner, but this contrasts with the experience of most neurologists and neurosurgeons who, similarly questioned, would instance meningiomas, angiomas, and occasionally neurofibromas.

It is not widely appreciated, however, that such symptoms may show complete remission following delivery, or, that in the case of certain slowly growing tumours, this sequence of events may recur repeatedly over a very long period, giving rise to considerable confusion in diagnosis.

Two striking cases have come under our care at the Midland Centre for Neurosurgery within a few months of each other, in each of which the type and position of the tumour was almost identical. These are recorded below and on extending this study to include other published reports of confirmed intracranial tumours showing repeated relapses and remissions during and after pregnancy, there is found a remarkable uniformity in the clinical picture, the histology, and the site of growth of the tumour.

\section{Case Reports}

Case 1.-A 45-year-old woman was referred complaining of impaired vision in the right eye. Sixteen years earlier, three weeks before the end of her fifth pregnancy, she had developed, quite painlessly, over a period of about three days, complete ptosis of the right eyelid and impaired movement of that eye. This remained until four weeks after delivery and then recovered completely. Five years later, a few weeks before the end of her sixth pregnancy, she again developed right-sided ptosis and partial oculomotor paresis, and again this recovered completely after delivery. Four

\footnotetext{
* From a paper read to the Association of British Neurologists at
} Belfast, April, 1957. years later still, three weeks before the end of her seventh pregnancy, exactly the same thing occurred, but recovery after delivery was only partial, and though the ptosis disappeared, she continued to have varying diplopia over the next eight years. During these years the ptosis frequently became complete again three days before menstruation was due and recovered during the week of menstruation. In the last 12 months her vision had begun to fail steadily and it was this that brought her under our care. The whole 16-year course had been completely painless.

Examination revealed that vision in the right eye was reduced to finger counting as a result of a large centrocaecal scotoma, and there was papilloedema in that eye. There was a partial right third nerve paresis, with total loss of upward ocular movement, but no other abnormal neurological signs.

Radiographs of the skull showed a thickening of the inner end of the right sphenoidal wing and there was a marked difference in the bone density surrounding the optic canals. A diagnosis of sphenoidal wing meningioma was made and a right carotid arteriogram carried out which did not show any definite tumour vascularity, but the carotid artery was most severely constricted intracranially over several millimetres (Fig. 1).

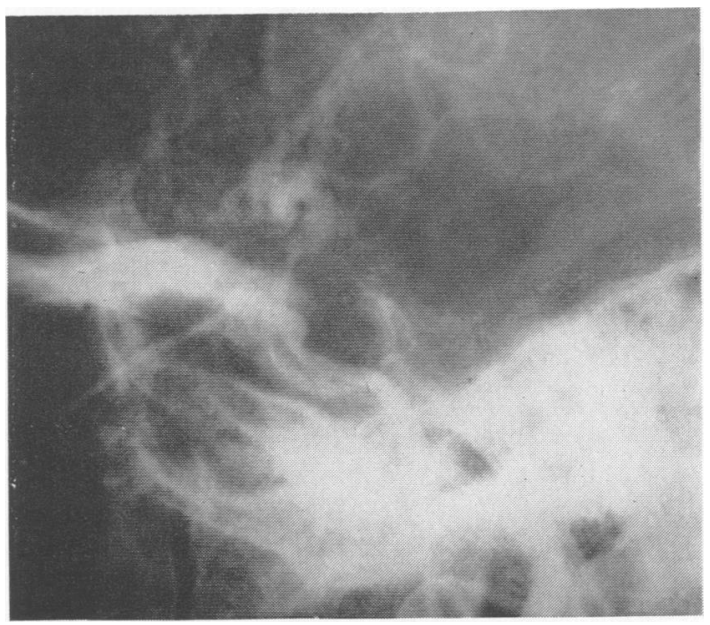

Fig. 1.-Enlargement of an arteriogram of Case 1 showing gross constriction of the carotid artery in its intracranial course. 
At exploration a meningioma-en-plaque was discovered extending over the sphenoidal wing, encircling the optic nerve, and there was a tongue of tumour extending backwards to encircle and constrict the carotid artery. As much tumour as could be seen was removed and the optic nerve and the carotid artery were freed; a course of deep $x$-ray therapy was given, and two and a half years later the patient is symptom free, there is no papilloedema, and vision is better than 6/24 in that eye.

Case 2.-A 37-year-old woman in the 36th week of her third pregnancy had experienced diplopia for four weeks and numbness over the right side of the face for two weeks. Her first attacks of diplopia, 14 years earlier, had many times occurred in the pre-menstrual week and recovered during the period of menstruation. In the intervening years she had had two previous pregnancies, and in each of these, a few weeks before she was due for delivery, she had developed severe diplopia, which had recovered completely within four weeks after parturition. Her present attack was the worst, and this was the first time she had experienced numbness of the face. The course of the disease had been entirely painless.

On examination there was a mild right-sided exophthalmos and paralysis of the right third nerve. Sensation to pain was lost over the first and second divisions of the fifth. nerve and impaired over the third division. Radiographs of the skull showed thickening of the inner end of the sphenoidal wing on the right and the bone surrounding the optic canal was thickened on that side.

Her pregnancy was allowed to proceed to term, and after delivery the sensory loss practically disappeared and the diplopia considerably improved.

A carotid arteriogram, six weeks after delivery, again showed little if any tumour circulation, but a curious, fusiform constriction of the intracranial portion of the artery (Fig. 2).

On exploration, a meningioma-en-plaque was found to spread over the sphenoidal wing and a thin layer of tumour extended backwards, compressing the carotid

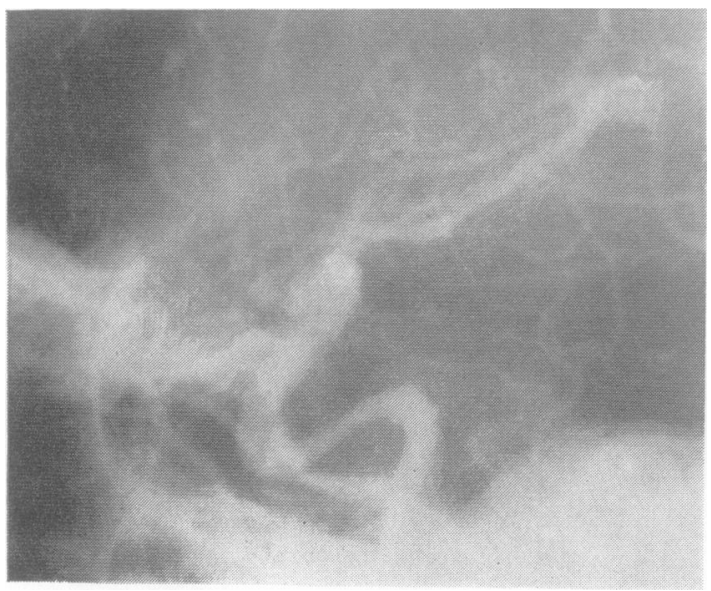

Fig. 2.-Enlargement of an arteriogram of Case 2 showing fusiform narrowing of the carotid syphen. artery, and extending into Meckel's cave. The tumour was widely excised and the carotid artery freed. Following operation, the patient has a permanent ptosis and strabismus, which are being corrected by plastic procedures. She has otherwise remained well for two years.

\section{Discussion}

Though study of the literature reveals many reports and references regarding all types of intracranial tumour whose growth appears to have been stimulated by pregnancy, when one extracts those cases in which symptoms have completely recovered after delivery, and, in particular, those in which this sequence of events has been repeated in two or more pregnancies, a very different picture emerges.

Fischer in 1931 described a woman who developed कै failing vision in her first pregnancy. Vision re- $\vec{\circ}$ covered completely after delivery, but progressed again to total blindness in the seventh month of a subsequent pregnancy, again completely recovering after delivery. In 1935 he reported further on the same patient, who by that time had been discovered is to have a parasellar meningioma. Hagedoorn (1937) $\vec{i}$ discussing relapsing visual field changes of unknow cause during pregnancy, mentions a 36-year-ole woman who developed a central scotoma in the right eye in the fifth month of her tenth pregnancy $\overrightarrow{2}$ which recovered completely after delivery, but recurred at the same stage of the next pregnancye This was terminated and vision again recovereci It later deteriorated and she was found to have $\infty$ large suprasellar meningioma. Walsh (1947) includes a case in which a central scotoma developed towards the end of a third pregnancy, recovered after delivery, and recurred two years later, when a meningioma was excised from the inner third of the sphenoidal wing. Rand and Andler (1950) described a patient who developed ptosis, exophthalmos, and visual failure towards the end of each of two pregnancies with recovery after delivery and later was found to have a sphenoidal wing meningioma. Finally, Weyand, MacCarty, and Wilson (1951), discussing the influence of pregnancy on meningiomas around the optic chiasma, include five cases showing two or more relapses and recoveries during and after pregnancy, each of which was found to have a suprasellar meningioma. They stress the fact that the symptoms developed in the later months. One of their cases also had attacks of blurred vision preceding menstruation, as in our cases.

Including our cases there are, therefore, on record 11 examples of meningiomas showing development and complete remission of symptoms during and after two or more pregnancies, and in each case the meningioma has been described as suprasellar, para- 
sellar, or medial sphenoidal wing. In every case it has been notable that headache or supra-orbital pain has been minimal or absent and that the symptoms have developed during the last four months of pregnancy. As the symptoms have been central scotomata, diplopia, or unilateral ptosis, it is not difficult to imagine that disseminated sclerosis has often been diagnosed and myasthenia gravis or even aneurysms have been considered.

Explanations of varied complexity have been offered for this sequence of events, ranging from the view that parasellar tumours might be particularly sensitive to hormonal influences, to a very much more simple suggestion of a purely mechanical nature, and it is this that seems most likely. Accepting the fact that any meningiomas may increase in size during pregnancy, the particularly intimate relationship borne by these tumours to the optic and oculomotor nerves is such that only a small increase in size is required to produce quite a dramatic increase in symptoms. It is notable that spinal tumours may behave in a similar manner, room for expansion again being limited. In contrast, convexity meningiomas may enlarge considerably without any striking new manifestations.

Again, various explanations have been offered for the actual increase in size that takes place during pregnancy, but the most likely is that, as a part of the general water retention in the body, the fluid content of the tumours is increased. Some support is given to this hypothesis by Weyand, MacCarty, and Wilson (1951), who twice removed meningiomas during the course of one of the pregnancies in which there had been symptomatic relapse, and the cytology was rather different from that usually seen. The cells were markedly swollen and the cytoplasm had a curious foamy appearance, but there was no increase in the degree of cellularity or in mitotic figures. The tumours appeared to be swollen, but the cells were not multiplying, so that a reduction in size at a later date could be quite easily understood. As a result of this study, we wish to suggest that if a patient develops unilateral scotomata or oculomotor defects during the latter half of pregnancy, particular attention should be paid to the possibility that she is harbouring a sphenoidal wing, parasellar, or suprasellar meningioma; that such a patient should be carefully investigated with this in mind and that if these investigations give the slightest suggestion that such a tumour is present, the area should be explored. This is neither a difficult nor a dangerous procedure and not only may the vision of an eye be saved if the diagnosis is established during an early relapse (a result often not achieved later, even after successful removal of the tumour), but also complete carotid strangulation, which in our cases was imminent, may be prevented. Hemiplegia may thus be avoided, even if radical removal of the tumour results in severe damage to the oculomotor nerve.

\section{Summary}

Meningiomas in the parasellar situation may produce relapse and remission of symptoms in relation to pregnancy and the menses over many years. Such symptoms-painless unilateral scotomata or oculomotor palsies-can cause serious diagnostic errors.

Two illustrative cases are described and nine others have been previously reported. The explanation of this behaviour is considered and the importance of early exploration stressed.

\section{REFERENCES}

Fischer, F. (1931). Z. Augenheilk., 75, 343. (1935). Ibid., 85, 88.

Hagedoorn, A. (1937). Amer. J. Ophthal., 20, 690.

Rand, C. W., and Andler, M. (1950). Arch. Neurol. Psychiat. (Chicago) 63, 1.

Walsh, F. B. (1947). Clinical Neuro-Ophthalmology, p. 1161. Williams and Wilkins, Baltimore.

Weyand, R. D., MacCarty, C.S., and Wilson, R. B. (1951). Surg. Clin. N. Amer., 31, 1225 . 